\title{
Age-Induced Aortic Modifications Are Accompanied By Alteration in the Antioxidant Defense System in Female Rats
}

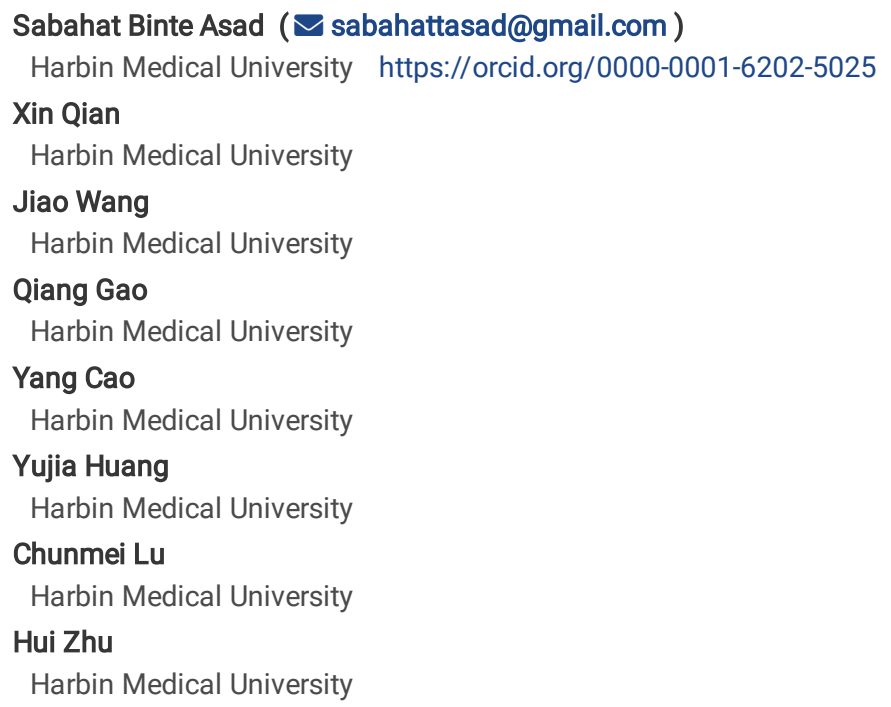

\section{Research Article}

Keywords: aging, blood pressure, aorta, antioxidant defense system, NRF2/KEAP-1 pathway

Posted Date: October 25th, 2021

DOI: https://doi.org/10.21203/rs.3.rs-1002842/v1

License: (ㄷ) (i) This work is licensed under a Creative Commons Attribution 4.0 International License. Read Full License 


\section{Abstract}

Aging causes significant structural and functional changes in the vasculature, disrupting homeostasis and affecting the cardiovascular system's performance. This study looked into the effects of aging on the aorta and how that affects antioxidant capability. As a result, healthy aging female SD rats $(n=10)$ were compared to their younger counterparts. Blood pressure, serum lipid profile, aortic histology examination, and the expression of antioxidant enzymes were all assessed. When aged rats were compared to young rats, their blood pressure and serum lipid levels were dramatically different. Furthermore, histological examination of the aorta of aging rats revealed structural changes, characteristic apoptotic nuclear morphology, and the formation of fatty deposits. Different antioxidant enzyme's protein expression was also studied. In aged rats, the expressions of SOD-1(superoxide dismutase), GSS(glutathione synthetase), NRF2(NF-E2-related factor 2), KEAP-1(Kelch-like ECH-associated protein 1), and HO-1(Heme oxygenase 1) were drastically changed. This study revealed that age-induced aortic changes are associated with an altered antioxidant system, according to this study.

\section{Introduction}

As an unavoidable aspect of life, aging is a composite natural process of becoming older. Aging introduces progressive structural and functional deterioration in almost all tissues, resulting in the gradual decline of the body's ability to maintain homeostasis, leading to natural death [1]. Vascular aging poses the highest risk for cardiovascular pathologies, and the aorta is considered a sensitive organ affected by aging [2]. Even with nonpathological aging, the aorta undergoes many structural distortions such as enlarged lumen, intimal-medial thickening (vascular remodeling), increased collagen and decreased elastin contents, and thin and fragmented elastic lamina in both human and animals [3, 4]. Besides, age-associated genderbased differences in aortic structure and function have also been discovered [5]

Previous studies have highlighted the association between aging and apoptosis [6]. Generally, during aging, the rate of apoptosis increases in many tissues and organ systems, including the cardiovascular system, endocrine system, immune system, and nervous system [7, 8]. Earlier, aging studies have documented the link between aortic stiffness and elevated blood pressure [9]. Likewise, age-related elevation in cholesterol levels has also been reported. With aging, blood lipid contents, especially LDL level increases, accounting for the accumulation of lipids in the vascular tissues, resulting in the atherogenic burden [10].

Antioxidants are scavenger molecules that can slow or prevent the oxidation process by neutralizing the deleterious effects of ROS. Under typical physiological conditions, there is an equilibrium between oxidant production and their elimination by the antioxidative system to prevent oxidative stress and sustain homeostasis [11]. Oxidative stress is considered a signature feature of aging caused by an imbalance between the oxidant/antioxidant equilibrium. Aerobic organisms are well-equipped with the cellular defensive antioxidant system to protect cells from oxidative insult [12, 13]. The antioxidant capacity comprises first-line defense antioxidants such as SOD-1, CAT, glutathione system, phase II detoxifying enzymes such as NQO-1 and HO-1, and different cytoprotective signaling pathways such as NRF2/KEAP-1 pathway. The antioxidant defense system plays a central role in cellular protection by eliminating harmful oxidants and preventing cells from oxidative damage [12].

The superoxide SOD-1 rapidly converts superoxide anion $\left(\mathrm{O}_{2}^{-}\right)$to hydrogen peroxide $\left(\mathrm{H}_{2} \mathrm{O}_{2}\right)$ and oxygen $\left(\mathrm{O}_{2}\right)$. Subsequently, hydrogen peroxide is decomposed into water and oxygen by the CAT enzyme [11]. One of the under-explored enzymes is glutathione synthetase (GSS), a pivotal contributor in the glutathione synthesis pathway. GSS indirectly plays an antioxidant role by mediating the 2nd step in the synthesis of GSH [13]. NRF2 is a redoxsensitive transcription factor that facilitates the transcription of many antioxidants via binding with a particular antioxidant response element (ARE) in that gene's promoter. Under normal conditions, NRF2 activity is tightly regulated by its cytoplasmic inhibitor KEAP-1 [14, 15]. During oxidative stress, the KEAP-1 undergoes conformational changes. Consequently, NRF2 dissociates from KEAP-1, translocates into the nucleus, and regulates the cytoprotective phase II detoxifying enzymes such as NQO-1 and HO-1 [15].

Numerous aging studies report that the decline in the antioxidant capacity results in oxidative stress-related cellular damage in different tissues [12]. Nevertheless, the mechanism of oxidative cellular damage in the aorta in response to the altered antioxidant system is not fully explored. This research was intended to explore the relationship between age-mediated aortic changes and the antioxidant defense system. In this context, blood pressure and serum lipid levels, aortic histological analysis, and the expression of different antioxidant proteins were evaluated to understand their correlation with the ageing process's complexity in the aortic wall.

\section{Materials And Methods}

\subsection{Animals}

Twenty female Sprague-Dawley (SD) rats were acquired from the animal center of Harbin Medical University. Rats were divided into two groups ( $n=10 /$ group): healthy young (3 months old) and aging (24 months old). Physiological aging symptoms were confirmed via vaginal smears exhibiting the disorder of the estrous cycle with low levels of senile estrogen. All rats were maintained under controlled conditions: (temperature $\left(22-25^{\circ} \mathrm{C}\right)$, humidity (50 $\pm 10 \%$ ), and a 12-h dark/light cycle), and received the standard chow diet ad libitum with free access to water. All experimental protocols used in this research were approved by the Animal Care and Use Committee (IACUC) of Harbin Medical University, China.

\subsection{Blood pressure}


A week before sacrificing the animals, blood pressure was measured non-invasively in conscious rats by tail and cuff detector (BP-2010E, Softron biotechnology Ltd. China). Briefly, each rat's tail was heated to $37^{\circ} \mathrm{C}$ for $10 \mathrm{~min}$, and the cuff with a pneumatic pulse sensor was placed around the animal's tail. Systolic, diastolic, and pulse pressure measurements were recorded in each rat and then averaged.

\subsection{Sample collection}

All animals were anesthetized with the intraperitoneal injection of $10 \%$ chloral hydrate and euthanized. Blood samples were collected and placed at $4^{\circ} \mathrm{C}$ for 1 hour and then centrifuged at $3000 \mathrm{rpm}$ at $4^{\circ} \mathrm{C}$ for $15 \mathrm{~min}$. The collected serum samples were stored at $-80^{\circ} \mathrm{C}$ for further evaluation. The thoracic aorta was excised, and all adventitial fats were removed. A part of the aorta was fixed in $4 \%$ paraformaldehyde and embedded in paraffin, and the remaining tissues were flash-frozen in liquid nitrogen and then stored at $-80^{\circ} \mathrm{C}$ for further analysis.

\subsection{Serum lipid analysis}

Serum levels of triglyceride (TG), total cholesterol (TC), low-density lipoprotein (LDL), and high-density lipoprotein (HDL) were evaluated using the HDL and LDL Quantification Fluorometric kit (Nanjing Jiancheng Bioengineering Institute, China).

\subsection{Histological analysis by H\&E staining}

The paraffin-embedded tissues were cut cross-sectionally into $5 \mu \mathrm{m}$ thick sections by Leica RM 2016 rotator microtome. According to the protocol described previously, the paraffin sections were deparaffinized, rehydrated, and then stained with hematoxylin and eosin (H\&E) [16]. H\&E stained slides were observed under a light microscope, and photographs were taken using the M8 Microscope \& Scanner (Precipoint, German). Images were processed with ViewPoint BETA v1.0.0.0 software. Aortic wall thickness was calculated using Image J software.

\subsection{Nuclear morphological analysis by Hoechst 33258 staining}

Aortic tissues were stained with Hoechst 33258 fluorescent dye (Beyotime, China) to observe apoptotic nuclear morphological changes. Briefly, the paraffin sections of aortic tissues were deparaffinized in xylene, rehydrated in gradient alcohol, washed twice with cold PBS, and then incubated with Hoechst 33258 for $10 \mathrm{~min}$ at room temperature in the dark. The sections were rewashed in PBS and examined under a fluorescence microscope (40x), and photographs were taken for further quantitative analysis. The percentage of apoptotic nuclei was calculated by counting the nuclei with apoptotic morphology among the normal nuclei.

\subsection{Oil Red 0 staining (ORO)}

In order to examine the aortic lipid contents, the freshly frozen (liquid nitrogen) aortic tissue samples from both groups were stained with Oil red 0 (ORO) (Nanjing Jiancheng Bioengineering Institute, China), as previously described [17]. The ORO-stained slides were examined under a light microscope and were photographed. The ORO staining intensity was quantified using ImageJ software.

\subsection{Western blotting}

The frozen aortic tissues were lysed with RIPA buffer, and total protein extracts were obtained by spinning at $14000 \mathrm{rpm}$ for 15 min. Total protein concentration was calculated using a bicinchoninic acid (BCA) protein assay kit (Thermo Scientific, USA). Total protein (20 $\mu$ g) were electrophoretically fractionated by $10 \%$ SDS-PAGE and transferred to a polyvinylidene difluoride (PVDF) membrane with a Trans-Blot SD semi-dry transfer cell (Bio-Rad Laboratories, Richmond, Calif.). The membranes were blocked with $5 \%$ non-fat milk dissolved in Tris-buffered saline with $0.1 \%$ Tween 20 for 2 hours. Next, membranes were incubated with the primary antibodies of $\beta$-actin (1:2000 dilution, Santa Cruz, USA), SOD-1(1:1000 dilution, Santa Cruz, USA), CAT(1:1000 dilution, Santa Cruz, USA), GSS (1:1000 dilution, Santa Cruz, USA), NRF2 (1:200 dilution, Abcam, UK), KEAP-1(1:200 dilution, Abcam, UK), NQ01(1:500 dilution, Abcam, UK), and HO-1(1:500 dilution, Abcam, UK) overnight at $4^{\circ} \mathrm{C}$. The subsequent day, membranes were incubated with horseradish peroxidase (HRP)-conjugated goat anti-rabbit IgG antibody (Santa Cruz Biotechnology, Santa Cruz, CA, USA) for 1 hour (room temperature). The blotted proteins were visualized using Pierce ECL Western Blotting Substrate (Engreen Biosystem, China). The relative density of bands was analyzed by ImageJ software.

\subsection{Immunohistochemistry (IHC)}

The Paraffin-embedded sections of the aorta were deparaffinized and rehydrated, then treated with $0.3 \% \mathrm{H}_{2} \mathrm{O}_{2}$ to block endogenous peroxidase activity. After blocking nonspecific reactions with goat serum, the primary rabbit polyclonal antibodies of SOD-1(1:500 dilution, Santa Cruz, USA), CAT(1:500 dilution), GSS(1:500 dilution), NRF2(1:200 dilution), KEAP-1(1:200 dilution,), NQ01(1:200 dilution), and H0-1(1:200 dilution) were added and incubated for 12 hours at $4^{\circ} \mathrm{C}$. The following day, after rinsing with PBS, the sections were incubated with biotinylated goat antibody to rabbit-rat lgG at $37^{\circ} \mathrm{C}$ for 1 hour and stained with DAB chromogenic and counterstained with hematoxylin. IHC stained slides were examined under a light microscope, and photographs were taken using the M8 Microscope \& Scanner. The IHC staining intensity of each IHC image was quantified using ImageJ software with the IHC profiler plugin [18], and the IHC optical density score was calculated as recommended by Seyed Jafari et al. [19].

\subsection{Statistical analysis}

All results in this study were expressed as mean \pm SD. For the statistical analysis, Student's t-test was applied for the group comparisons and was evaluated by GraphPad Prism version 6.0c (GraphPad, La Jolla, CA). P value less $5(p<0.05)$ was considered statistically significant.

\section{Results}




\subsection{Aging increases blood pressure in rats}

Systolic blood pressure (SBP), diastolic blood pressure (DBP), and pulse pressure (PP) were analyzed and compared between young and aging rats. SBP, DBP, and PP were significantly elevated in aging rats compared to those of young rats (Fig. 1a, b \& c). These results provide evidence that blood pressure increases with physiological aging.

\subsection{Aging regulates serum lipid profile}

As compared to young rats, the serum HDL levels significantly decreased, whereas LDL levels remarkably increased in the aging rat (Fig. 2a\&b). Although the total cholesterol and triglycerides levels slightly decreased in aging rats, they were not statistically significant (Fig. 2c \& d).

\subsection{Aging alters the histology of the aorta}

Histological examination of the aorta from young rats expressed typical morphology. The tunica intima (TI) was very thin and composed of a continuous layer of thin flattened endothelial cells with flattened nuclei, and the luminal surface of the aorta was very regular and smooth. The tunica media (TM) was characterized by numerous diverse elastic laminae, which were wavy, parallel, and regularly arranged. Smooth muscle cells (SMCs) with oval to flattened nuclei were regularly arranged in the narrow spaces between the concentric lamellae. The tunica adventitia (TA) was comprised of loose connective tissue (Fig. 3a).

In contrast, however, the aorta of aging rats revealed noticeable destructive morphology. TI was thick to the extent that it could easily be distinguished from the TM. The endothelial lining was featured with endothelial cell proliferation as their nuclei were close to each other with the invasion of the subendothelial connective tissues, and the luminal surface was relatively rough and irregular. TM was particularly thick with an increased number of elastic lamellae. The elastic fibers of the TM were thin and remarkably spacious, with some level of fragmentation. SMCs were also proliferating in between the elastic laminae and showed binucleate and multinucleate appearance. The TA was thickened and showed fibrosis (Fig. 3b). Aortic wall thickness showed a significant increase in aging rats compared to young rats (Fig. 3c).

\subsection{Aging alters nuclear morphology}

Nuclear morphological changes were analyzed by Hoechst 33258 staining. As shown in Fig. 4a, the morphology of the nucleus was normal and intact and displayed fewer apoptotic nuclei in the young rats. In contrast, in aging rats, it was observed that nuclear staining exhibited typical apoptotic morphological changes such as chromatin condensation, nuclear fragmentation, and high apoptotic bodies (Fig. 4b). The percentage of apoptotic nuclei in the aging rats was significantly higher than that of the young rats (Fig. 4c).

\subsection{There is the accumulation of lipid in the aortic wall during aging}

The ORO staining was performed to visualize the quantity and intracellular localization of neutral triglycerides, lipid, and fat contents in the aorta. As illustrated in Fig. 5a, young rats displayed very weak ORO lipid staining as very few lipid droplets were seen in the aortic wall. By comparison, aging rats exhibited highly positive ORO staining in the aorta. Microscopically, the lipid droplets appeared as streaks of small red granules or grape-like clusters in all three layers. Besides, some enlarged lipid droplets were also detected within some regions in tunica media and tunica adventitia, representing the high amount of fat deposition in that particular region (Fig. 5b). The ORO positive area percentage was significantly increased in the aorta of aging rats (Fig. 5c).

\subsection{Altered expression of antioxidant proteins with aging in the aorta}

To investigate the effect of aging on the aorta's antioxidant enzymes, we analyzed protein expression levels of SOD-1, CAT, GSS, NRF2, KEAP-1, HO-I, and NQO-1 by western blot. Western blot results revealed that, compared to young rats, the protein expression levels of SOD-1 and GSS decreased significantly, whereas the protein expression levels of NRF2, KEAP-1, and HO-1 proteins significantly increased in aging rats. However, the protein expression of CAT was slightly decreased, and the protein expression of NQO-1 was slightly increased in aging rats compared to young rats (Fig. 5a\&b).

Immunohistochemistry (IHC) was performed to visualize antioxidant proteins' expression in the aorta from young and aging rats. IHC results confirmed the western blot findings and revealed that the protein expression levels of SOD-1 and GSS decreased significantly in aging rats. However, quite the opposite, the immunostaining for NRF2, KEAP-1, and HO-1 increased significantly in aging rats' aorta compared to the young rats. Notably, immunostaining for CAT and NQO-1 was weak in both groups, and no noticeable difference was observed between young and aging rats (Fig. 7a\&b). Quantitative assessments of the staining confirmed that the mean optical intensity density (MOID) score for SOD-1 and GSS showed a significant decrease, whereas the score for NRF2, KEAP-1, and HO-1 showed a significant increase in aging rats relative to the younger group. The MOID score for CAT and NQO-1 did not differ significantly in both groups (Fig. 7c).

\section{Discussion}

The present study evaluated the association between vascular aging and the antioxidant defense system by investigating age-induced alteration in blood pressure, serum lipid levels, histological analysis, and antioxidant capacity in the aorta of young and aging rats.

Various studies have reported the rise in blood pressure that is considered an unavoidable part of healthy aging and predominantly accompanied by structural alterations in the arteries [20]. Our results showing significant elevation in the SBP, DBP, and PP in our aging model agrees with previous data. Additionally, previous data reported that high blood pressure is associated with loss of balance between oxidants and antioxidants [21]. Notably, the 
correlation between the age-associated decline in circulating sex hormones and elevated blood pressure has also been reported [22]. A gradual loss of gonadal hormones such as estrogen with aging has also been reported to play a role in blood pressure elevation in aging female rats. These findings and our data suggest that there is considerable correspondence between an age-related rise in blood pressure, which could result from aortic structural changes, and variation in antioxidant status.

It is well-known that aging affects normal cholesterol metabolism, resulting in an alteration in serum lipid profile [23]. This study detected significantly high serum LDL levels and significantly low serum HDL levels in aging rats than young rats. However, no significant change in the serum TG and TC levels was observed with aging. Consistent with our findings, age-related higher LDL and lower HDL levels have also been mentioned in previous reports $[24,25]$. Likewise, no significant change in TG and TC levels was observed between the 24-month-old and 10-month-old rats [26]. It is well acknowledged that high LDL/low HDL levels in blood account for the accumulation of fat in the aorta and increase atherosclerosis incidence [10]. We further examined whether these age-related alterations in serum cholesterol levels are associated with the accumulation of fat/lipid in the aorta. As expected, high lipid accumulation in the aged aorta was observed. Notably, no apparent atheromatous plaque was observed in the aorta from aging rats. These findings suggest that increased fat/lipid components in the aorta might be due to altered blood cholesterol levels and can be considered a normal part of aging, independent of atherosclerosis.

Previous studies have reported that aging brings about profound structural and histological alterations in the aortic wall [1,3]. By examining the histological changes in the aorta of young and aging rats using H\&E staining, the aortic wall's basic architecture was found to be altered in aging rats. By comparison, aorta obtained from young rats exhibited normal aortic morphology. These observations in the aorta of aging rats agree with previous reports using different aging models $[27,28]$. Consistent with our results, similar structural alterations in the aorta were observed in aged male albino rats [4]. In humans, age-associated modifications in the vascular structure have also been reported [29, 30]. We further investigated the effect of aging on nuclear morphology and apoptosis. Hoechst staining results revealed that aorta from aging rats exhibited typical apoptotic nuclear morphology such as blebbing, nuclear fragmentation, chromatin condensation, and cell shrinkage. The percentage of apoptotic nuclei was significantly higher in the aged aorta. These results agree with previously reported findings [6]. Importantly, increased apoptosis during aging is considered in one instance as a protective mechanism of the body against an accumulation of injurious defective cells and in another related to an age-related decline in the structure and functional integrity of various tissues [6].

Data from various studies have described that the accumulation of oxidative damage resulting from oxidant/antioxidant imbalance might play an essential role in structural alterations and increase apoptosis in various tissues with aging [6, 8]. These findings lead us to hypothesize that physiological aging accompanied by degenerative structural changes and increases apoptosis in the aorta could involve the oxidative mechanism. However, this underlying mechanism in the aged aorta is poorly understood.

Antioxidant enzymes protect the body against the deleterious effect of oxidative stress, and a decline in the antioxidant capacity results in the production of oxidative stress-related cellular damage in the tissues [12]. To evaluate the role of aging on the aorta's antioxidant capacity, we analyzed and compared some antioxidative and cytoprotective proteins' expression levels, i.e., SOD-1, GSS, CAT, NRF2, KEAP-1, HO-1, and NQO-1 in the aorta from young and aging rats. Western blot results and immunohistochemical analyses revealed that SOD-1 and GSS significantly decreased, and NRF2, KEAP-1, and HO-1 significantly increased in the aorta of aging rats in comparison with young rats. Additionally, the protein expression levels of CAT and NQO-1 remained unaltered. These results suggest that the age-related decline in the expression of SOD-1 and GSS could account for the oxidative stressinduced cellular damage to the aortic cells leading to the morphological and physiological alteration in the aorta with senescence. Similar to our findings, a study was performed in male Wister rats with ages ranging from 1 month to 24 months. The findings demonstrated an age-related decline in SOD-1 while CAT expression was unchanged in the aorta [31]. We could not find any research related to GSS alteration with aging in the aorta. However, few studies have reported changes in GSS protein expression in different organs with aging [32]. Surprisingly, the observed noticeable increase in the basal expressions of NRF2, KEAP-1, and HO-1 proteins in the aorta of aging rats is supported by others' findings on different organs other than the aorta [33]. L. Zhou et al. found that the basal expression of NRF2 regulated genes increased with aging in human bronchial epithelial cells, while inducible expression was declined in these cells [34]. Contrary to our results, impaired NRF2/KEAP-1 signaling and down-regulation of both NQO-1 and HO-1 with aging have been reported in various studies [35]. Z. Ungvari et al. reported that vascular oxidative stress is accompanied by a decreased expression of NRF2, NQO-1, and HO-1 in aged Fischer 344 _ Brown Norway rats [36]. A possible reason for this inconsistency might be using the different cells, tissues, and animals, and further investigations are needed to elucidate these controversial results.

Many studies have highlighted that, upon exposure of cells to oxidative stress, eukaryotic cells induce complex redox-sensitive NRF2/KEAP-1 signaling pathway to protect cells against oxidative injury and maintain cellular homeostasis [37]. NRF2/KEAP-1 signaling pathway up-regulates various antioxidants and phase II detoxifying enzymes such as HO-1 and NQO-1. HO-1 and NQO-1 are essential components of the cellular stress response and play a critical role in cellular protection against oxidative insult [35]. Importantly, NRF2 was mainly located in the nucleus of aortic cells, indicating the possible basal induction of NRF2/KEAP-1 signaling in the aorta in response to the occurrence of oxidative stress during aging. These findings suggest no cellular injury or oxidative damage in the aorta from young rats accounting for the basal levels of NRF2/KEAP-1 signaling pathway and its subsequent downstream regulation of NQO-1 and HO-1 proteins. However, in the aging rats, increased levels of NRF2 and KEAP-1 protein in the aortic tissues explained the age-associated overactivation of NRF2/KEAP-1 signaling pathway. Furthermore, the increased expression of HO- 1 in the aorta of aging rats further confirmed that the induction of this enzyme is governed by the activation of NRF2/KEAP-1 pathway in response to oxidative stress in the aortic tissues. Interestingly, we found that the expression of NQO-1 protein was not significant in the young and aging rats. It confers the notion that in the aging aorta, the transcriptional antioxidant response of NRF2/KEAP-1 pathway is involved in the up-regulation of HO-1 compared to NQO-1 in female SD 
rats. Further investigations are however required to fully elucidate the basic mechanism involved in the activation of the NRF2/KEAP-1 pathway and its regulation in the aged aorta using knockdown or overexpression studies.

In summary, it is believed that there is a substantial correlation between degenerative histological changes and decreased antioxidant capacity in the aorta during physiological aging. It is possible that the degenerative structural changes associated with aging are the result of oxidative stress caused by decreased tissue production of main antioxidants such as SOD-1 and GSS. In response, eukaryotic cells may activate NRF2/KEAP-1 signalling and increase HO-1 enzyme production as a compensatory strategy to preserve normal aortic function in the aged aorta, hence promoting healthy aging in the absence of antecedent pathological illness.

\section{Conclusions}

Aging may result in structural and functional changes to the aorta, as well as altered antioxidant defence system expression. These age-related changes in the antioxidant system of the old aorta may represent a compensation reaction designed to preserve normal homeostasis during aging.

\section{Declarations}

\section{Funding}

This work was supported by the grant from the Chinese National Natural Science Foundation (81571957), Heilongjiang Natural Science Foundation (QC2017089), Harbin Medical University graduate innovative research projects (YJSCX2017-2HYD, YJSCX2016-2HYD), and the Fundamental Research Funds for the Provincial Universities (2017-KYYWF-0297).

\section{Conflict of Interests}

The authors declare that they have no conflict of interest.

\section{Author Contributions:}

SB Conceptualization, Sabahat Binte Asad, Xin Qian, Hui Zhu and Chunmei Lu; Data curation, Sabahat Binte Asad and Qiang Gao; Formal analysis, Sabahat Binte Asad and Hui Zhu; Funding acquisition, Xin Qian, Hui Zhu and Chunmei Lu; Investigation, Sabahat Binte Asad, Xin Qian and Jiao Wang; Methodology, Sabahat Binte Asad, Xin Qian, Jiao Wang, Yang Cao and Yujia Huang; Project administration, Hui Zhu; Resources, Hui Zhu and Chunmei Lu; Software, Sabahat Binte Asad and Qiang Gao; Supervision, Hui Zhu and Chunmei Lu; Validation, Sabahat Binte Asad, Hui Zhu and Chunmei Lu; Visualization, Sabahat Binte Asad, Xin Qian, Jiao Wang, Qiang Gao, Yang Cao and Yujia Huang; Writing - original draft, Sabahat Binte Asad; Writing review \& editing, Sabahat Binte Asad and Hui Zhu.

\section{Ethical approval}

All experimental protocols used in this research were approved by the Animal Care and Use Committee (IACUC) of Harbin Medical University, China.

\section{Consent for participate}

Not applicable

\section{Consent for publication}

Not applicable

\section{References}

1. (2018) Aging related functional and structural changes in the heart and aorta: MitoTEMPO improves aged-cardiovascular performance. Exp Gerontol 110:172-181. https://doi.org/10.1016/j.exger.2018.06.012

2. Jani B, Rajkumar C (2006) Ageing and vascular ageing. Postgrad Med J 82:357-362. https://doi.org/10.1136/pgmj.2005.036053

3. Ivic I, Vamos Z, Cseplo P, Koller A (2017) From newborn to senescence morphological and functional remodeling leads to increased contractile capacity of arteries. Journals Gerontol - Ser A Biol Sci Med Sci 72:481-488. https://doi.org/10.1093/gerona/glw085

4. (2016) Histological Modifications Aging Aorta in Male Albino Rat. J Cytol Histol 07: https://doi.org/10.4172/2157-7099.1000408

5. (2014) Efficacy of Female Rat Models in Translational Cardiovascular Aging Research. J Aging Res 2014:1-14. https://doi.org/10.1155/2014/153127

6. Muradian K, Schachtschabel DO (2001) The role of apoptosis in aging and age-related disease: update. Zeitschrift fï ¿1/2r Gerontol Geriatr 34:441446. https://doi.org/10.1007/s003910170015

7. Qian X, Asad SB, Li J et al (2019) Link between cardiac function and the antioxidative defense mechanism in aged rats. Biochem Biophys Res Commun 513:1100-1105. https://doi.org/10.1016/J.BBRC.2019.03.182 
8. Lu B, Chen H-D, Lu H-G (2012) The relationship between apoptosis and aging. Adv Biosci Biotechnol 3:705-711.

https://doi.org/10.4236/abb.2012.326091

9. (2010) Hypertension in the elderly. J Nephrol 23 Suppl 1:S61-71

10. Wang JC, Bennett M (2012) Aging and Atherosclerosis. Circ Res 111:245-259. https://doi.org/10.1161/CIRCRESAHA.111.261388

11. Ighodaro OM, Akinloye OA (2017) First line defence antioxidants-superoxide dismutase (SOD), catalase (CAT) and glutathione peroxidase (GPX): Their fundamental role in the entire antioxidant defence grid. Alexandria J Med 1-7. https://doi.org/10.1016/j.ajme.2017.09.001

12. Kasapoglu M, Ozben T (2001) Alterations of antioxidant enzymes and oxidative stress markers in aging. Exp Gerontol 36:209-220

13. Espinosa-diez C, Miguel V, Mennerich D et al (2015) Redox Biology Antioxidant responses and cellular adjustments to oxidative stress. 6:183-197. https://doi.org/10.1016/j.redox.2015.07.008

14. Shih P-H, Yen G-C (2007) Differential expressions of antioxidant status in aging rats: the role of transcriptional factor Nrf2 and MAPK signaling pathway. Biogerontology 8:71-80. https://doi.org/10.1007/s10522-006-9033-y

15. Canning P, Sorrell FJ, Bullock AN (2015) Structural basis of Keap1 interactions with Nrf2. Free Radic Biol Med 88:101-107. https://doi.org/10.1016/J.FREERADBIOMED.2015.05.034

16. Andrés-Manzano MJ, Andrés V, Dorado B (2015) Oil Red O and Hematoxylin and Eosin Staining for Quantification of Atherosclerosis Burden in Mouse Aorta and Aortic Root. In: Methods in molecular biology (Clifton, N.J.). pp 85-99

17. Mehlem A, Hagberg CE, Muhl L et al (2013) Imaging of neutral lipids by oil red O for analyzing the metabolic status in health and disease. Nat Protoc 8:1149-1154. https://doi.org/10.1038/nprot.2013.055

18. Varghese F, Bukhari AB, Malhotra R, De A (2014) IHC Profiler: An Open Source Plugin for the Quantitative Evaluation and Automated Scoring of Immunohistochemistry Images of Human Tissue Samples. PLoS One 9:e96801. https://doi.org/10.1371/journal.pone.0096801

19. Seyed Jafari SM, Hunger RE (2017) IHC Optical Density Score. Appl Immunohistochem Mol Morphol 25:e12-e13. https://doi.org/10.1097/PAI.0000000000000370

20. Pinto E (2007) Blood pressure and ageing. Postgrad Med J 83:109-114. https://doi.org/10.1136/pgmj.2006.048371

21. Romero-Alvira D, Roche E (1996) High blood pressure, oxygen radicals and antioxidants: Etiological relationships. Med Hypotheses 46:414-420. https://doi.org/10.1016/S0306-9877(96)90196-6

22. (2004) Age-related decreases in gonadal hormones in Long-Evans rats: relationship to rise in arterial pressure. Endocrine 25:15-22. https://doi.org/10.1385/END0:25:1:15

23. Mesomya W, Hengsawadi D, Cuptapun Y (2001) Effect of Age on Serum Cholesterol and Triglyceride Levels in the Experimental Rats. 148:144-148

24. Ericsson S, Eriksson M, Vitols S et al (1991) Influence of age on the metabolism of plasma low density lipoproteins in healthy males. J Clin Invest 87:591-596. https://doi.org/10.1172/JCl115034

25. Schaefer EJ, Lamon-Fava S, Cohn SD et al (1994) Effects of age, gender, and menopausal status on plasma low density lipoprotein cholesterol and apolipoprotein B levels in the Framingham Offspring Study. J Lipid Res 35:779-792

26. Morisaki N, Fujiyama Y, Matsuoka N et al (1984) Role of aging in lipid metabolism in the aorta of rats. Gerontology 30:13-21. https://doi.org/10.1159/000212601

27. (2008) Aortic aging in the Fischer 344 / NNiaHSd x Brown Norway / BiNia Rat. J Pharmacol Sci 108:393-398. https://doi.org/10.1254/jphs.08R02CP

28. (2015) Age-Associated Alterations of Morphology and ProteinSignaling in the Female F344xBN Rat Aorta. J Gerontol Geriatr Res 04: https://doi.org/10.4172/2167-7182.1000196

29. (2007) Heart disease and stroke statistics-2007 update: a report from the American Heart Association Statistics Committee and Stroke Statistics Subcommittee. Circulation 115:e69-171. https://doi.org/10.1161/CIRCULATIONAHA.106.179918

30. (2011) Microscopic study of aorta in relation of different age groups: an observational study.

https://www.biomedscidirect.com/112/microscopic_study_of_aorta_in_relation_of_different_age_groups_an_observational_study/articlescategories. Accessed 15 Oct 2018

31. (2007) Loss of adaptation to oxidative stress as a mechanism for aortic damage in aging rats. J Physiol Biochem 63:239-247. https://doi.org/10.1007/BF03165787

32. (2015) Age-dependent changes of the antioxidant system in rat livers are accompanied by altered MAPK activation and a decline in motor signaling. EXCLI J 14:1273-1290. https://doi.org/10.17179/excli2015-734

33. Sachdeva MM, Cano M, Handa JT (2014) Nrf2 signaling is impaired in the aging RPE given an oxidative insult. Exp Eye Res 119:111-114. https://doi.org/10.1016/j.exer.2013.10.024

34. Zhou L, Zhang H, Davies KJA, Forman HJ (2018) Aging-related decline in the induction of Nrf2-regulated antioxidant genes in human bronchial epithelial cells. Redox Biol 14:35-40. https://doi.org/10.1016/j.redox.2017.08.014

35. (2015) Oxidative stress response and Nrf2 signaling in aging. Free Radic Biol Med 88:314-336. https://doi.org/10.1016/j.freeradbiomed.2015.05.036 
36. Ungvari Z, Bailey-Downs L, Sosnowska D et al (2011) Vascular oxidative stress in aging: a homeostatic failure due to dysregulation of NRF2mediated antioxidant response. AJP Hear Circ Physiol 301:H363-H372. https://doi.org/10.1152/ajpheart.01134.2010

37. Liguori I, Russo G, Curcio F et al (2018) Oxidative stress, aging, and diseases. Clin Interv Aging Volume 13:757-772. https://doi.org/10.2147/CIA.S158513

\section{Figures}

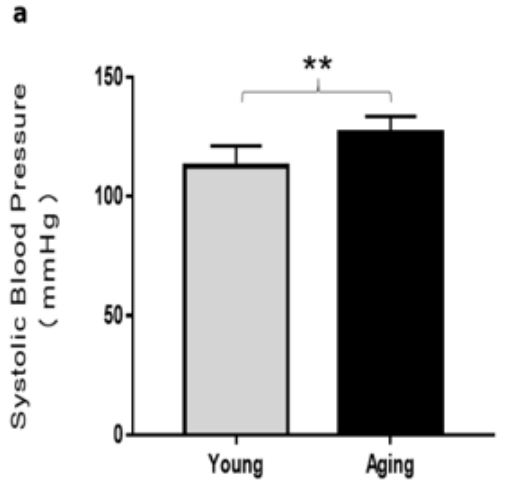

b

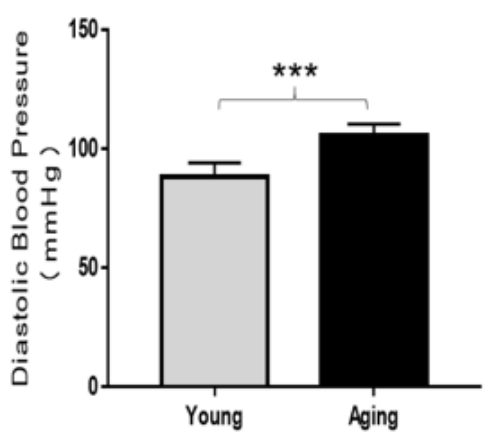

$c$

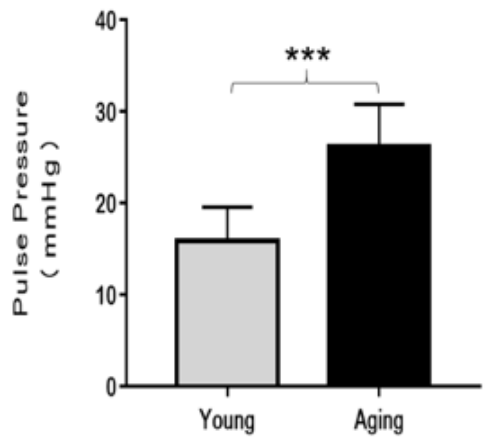

Figure 1

Comparison of blood pressure analysis between young and aging rats. In aging rats, systolic blood pressure (SBP), diastolic blood pressure (DBP), and pulse pressure (PP) significantly increased compared to the young rats. (a) Systolic blood pressure ** $(P<0.01)(b)$ diastolic blood pressure $\star \star \star ~(P<0.001)$, and (c) Pulse pressure ${ }^{\star \star \star}(P<0.001)$ vs. young rats. Data represent the mean $\pm S D$ of 10 rats.

a

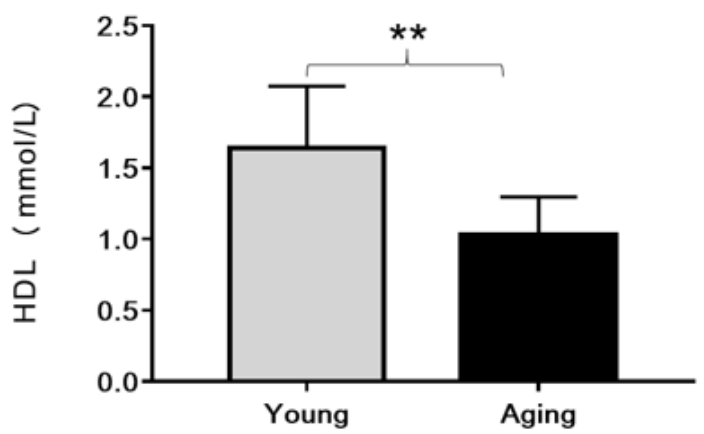

C

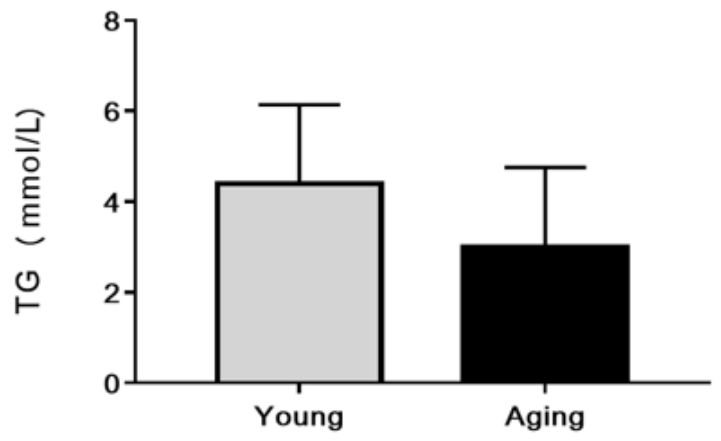

b
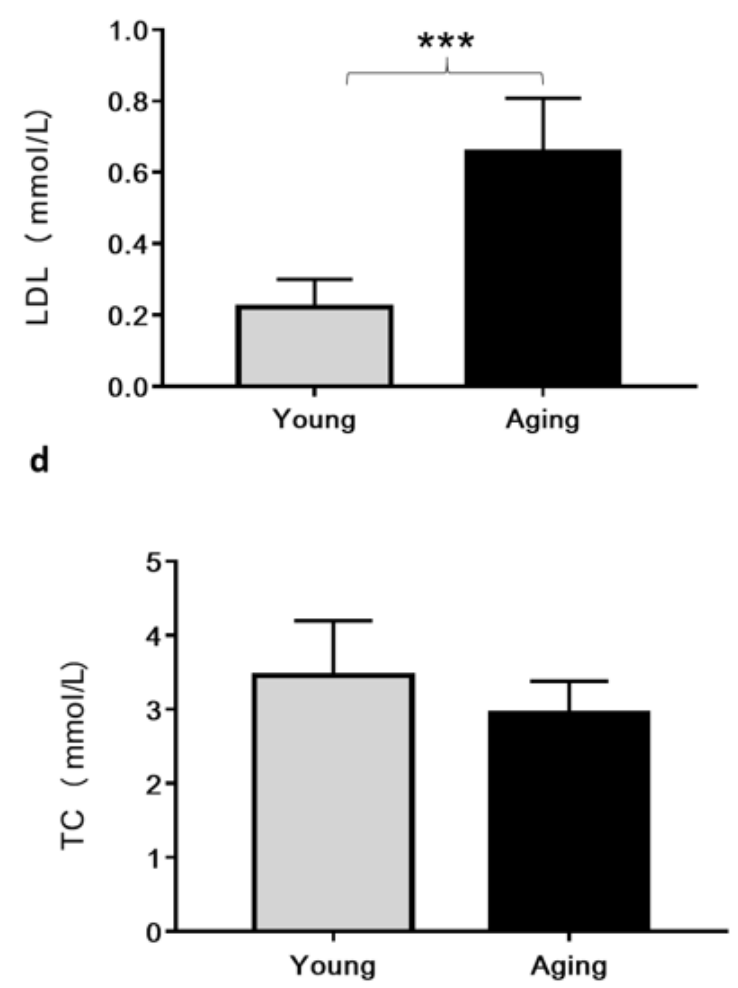

\section{Figure 2}

Comparison of serum lipid profile (mmol/L) between young and aging rats. Relative to young rats, (a) Serum HDL levels significantly decreased ** $(P<0.01)$ whereas (b) Serum LDL levels significantly increased in aging rats ${ }^{\star \star \star}(P<0.001)$. No significant differences were found in $(c)$ TG and $(d)$ TC 
levels between young and aging groups. Data represent the mean \pm SD. ( $n=10$ rats). Abbreviations: HDL=High-density lipoprotein, LDL= Low-density lipoprotein, TG=Triglyceride, and TC=Total cholesterol.
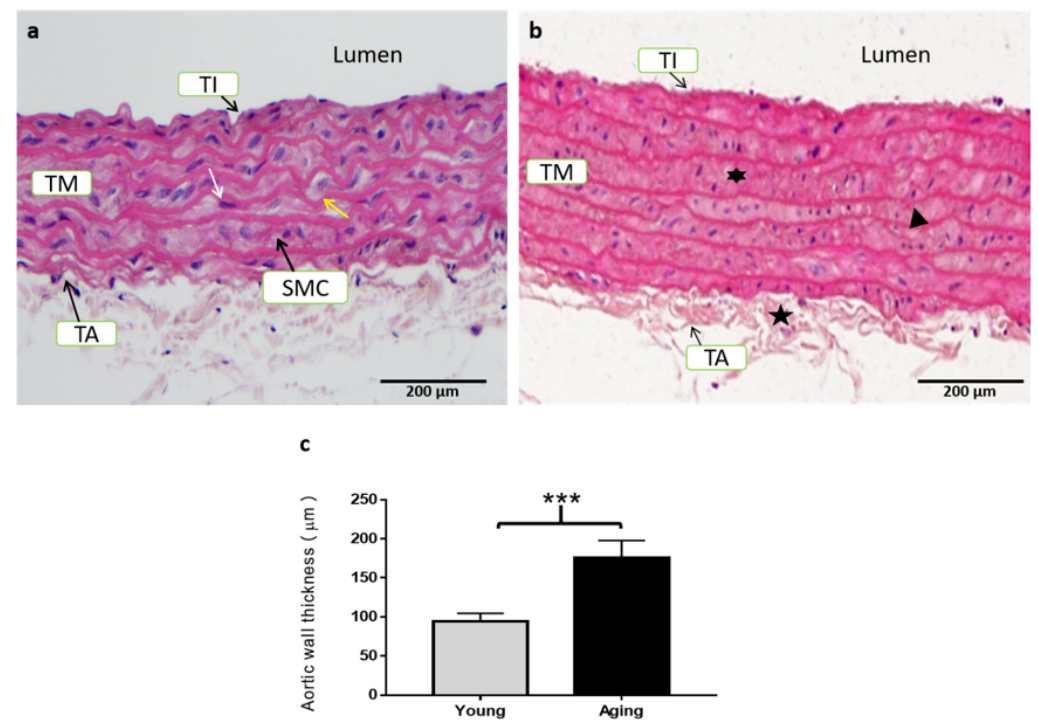

Figure. 3. Representative $H \& E$ staining of the aorta's cross-sectional tissue slices in

young and aging rats. (a) Histological examination of the aorta from young rats displayed normal morphology. (b) Histological examination of the aorta from aging rats showed degenerative morphological modifications in all three layers. Scale bar $=200 \mu \mathrm{m}$. (c) Aortic wall thickness was significantly increased in aging rats $* * *(P<0.001)$ vs. young rats. $(\mathrm{n}=3)$. Abbreviations: $\mathrm{TI}=$ Tunica intima, $\mathrm{TM}=$ Tunica media, $\mathrm{TA}=$ Tunica adventitia, $\mathrm{SMC}=$ Smooth muscle cells, IMT=Intimal-medial thickness, white arrow = internal elastic lamina, yellow arrow $=$ nucleus of smooth muscle cells, $\star=$ fibrosed tunica adventitia, $*=$ proliferation of smooth muscle cells, $\mathbf{\Lambda}=$ thin and fragmented elastic lamina.

\section{Figure 3}

See image above for figure legend. 


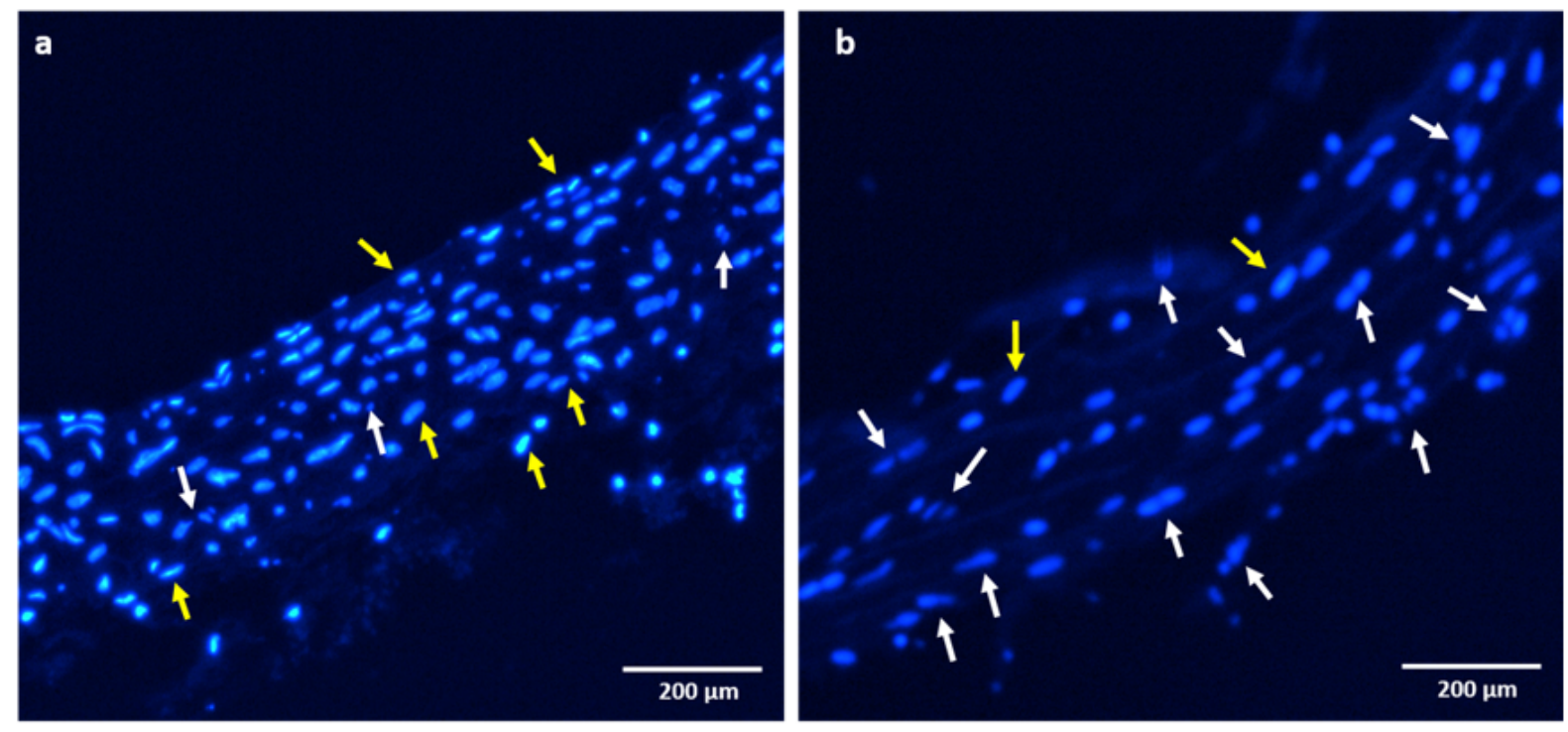

C

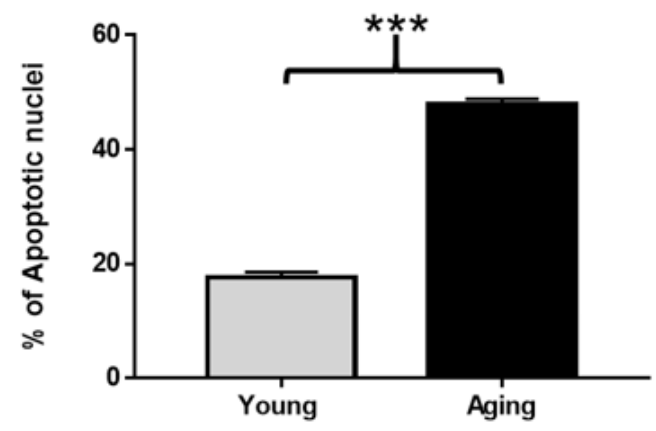

Figure 4

Assessment of nuclear morphological changes by Hoechst 33258 staining (a) Representative image showing normal nuclear morphology in young rats. (b) Representative image showing typical apoptotic nuclear morphology such as chromatin condensation, nuclear fragmentation, and increased number of apoptotic nuclei in aging rats. Scale bar $=200 \mu \mathrm{m}$. (c) \% of apoptotic nuclei significantly increased in aging rats $\star \star \star ~(P<0.001)$ vs. young rats. The yellow arrow points to normal nuclei, White arrow points to apoptotic nuclei. 

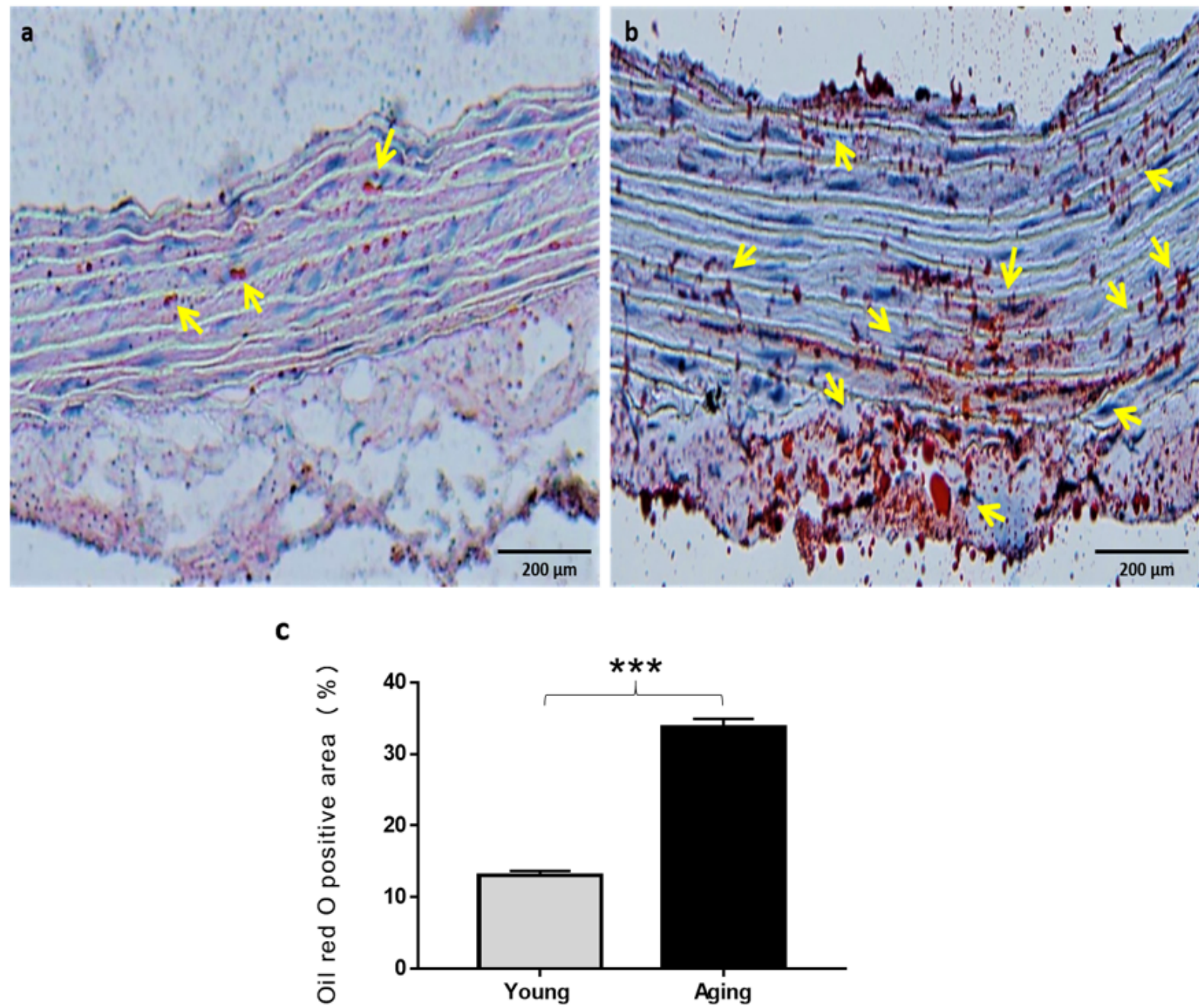

Figure 5

Oil red $\mathrm{O}$ staining of the aorta of the young and aging rats. (a) Representative images display weak ORO staining in the aorta of young rats (b) and high ORO staining in the aorta of aging rats that represents the accumulation of lipids in the aortic wall. (c) \% of ORO positive area significantly increased in aging rats $* \star \star ~(P<0.001)$ vs. young rats $(n=3)$. Abbreviations: ORO $=$ Oil red 0 , yellow arrow $=$ lipid droplets appeared as red granules in the aorta. Scale bar $=200 \mu \mathrm{m}$.

a

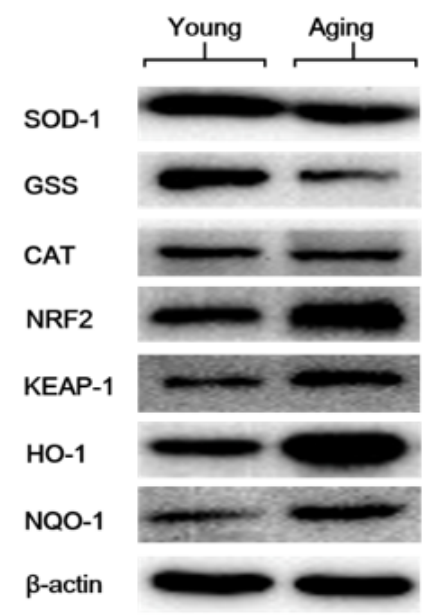

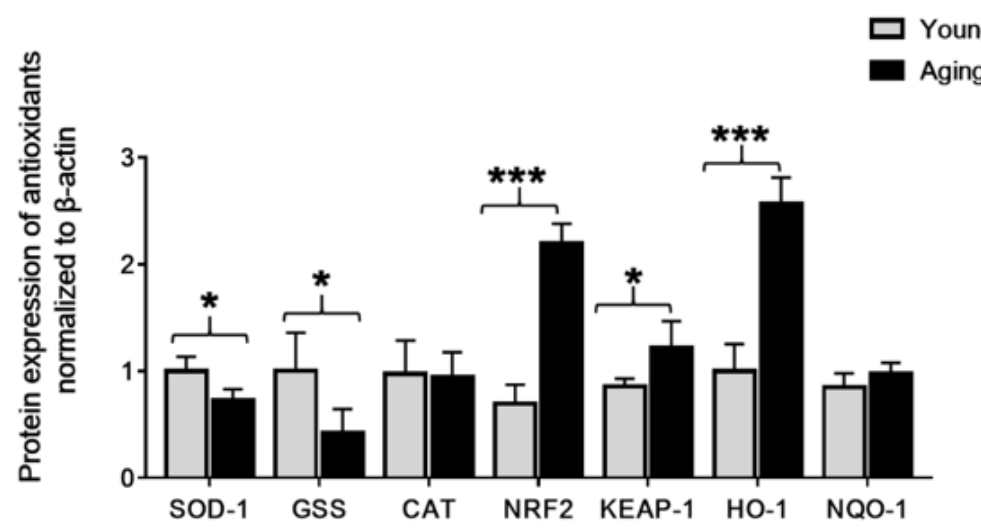

Figure 6 
The protein expression levels of SOD-1, GSS, CAT, NRF2, KEAP-1, HO-1, and NQ0-1 in the aorta of young and aging rats. (a) Representative immunoblot. (b) Densitometric analysis showing that the protein expression levels of SOD-1 and GSS significantly decreased * $(P<0.05)$, whereas NRF2, KEAP-1, and HO-1 significantly increased $\left(\star \star \star ~(P<0.001),{ }^{\star}(P<0.05)\right.$, ( ${ }^{\star * \star}(P<0.001)$ respectively) in the aging rats compared to the young rats. $\beta$-actin was used as an internal control. Abbreviations: SOD-1= superoxide dismutase-1, GSS= glutathione synthetase, CAT=catalase, NRF2= nuclear factor-erythroid 2-related factor-2, KEAP-1 = kelch-like ECH-associated protein, HO-1=heme oxygenase-1, and NQO-1=NAD(P)H: quinone oxidoreductase.

a

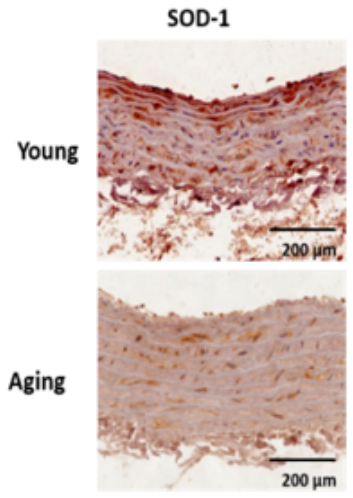

b

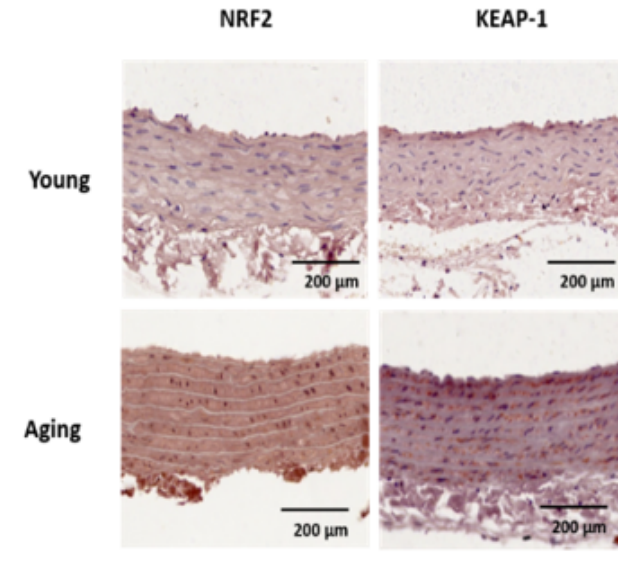

GSS

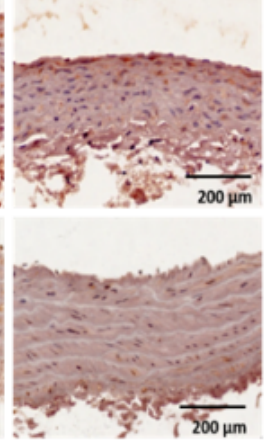

KEAP-1
CAT

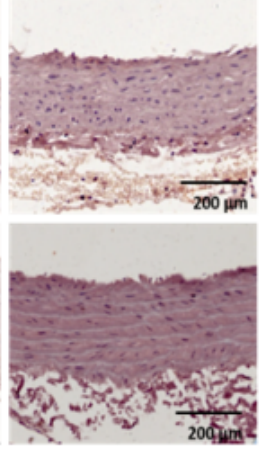

HO-1 c

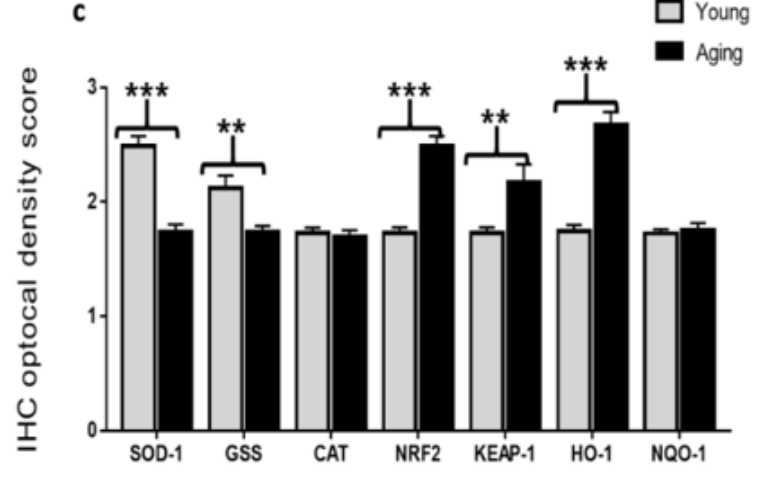

\section{Figure 7}

(a) Representative images of immunohistochemically stained slices for SOD-1, GSS, and CAT (b) as well as for NRF2, KEAP-1, HO-1, and NQO-1. The brownish color indicates positive immunostaining. Scale bar=200 $\mu \mathrm{m}$. (c) The mean optical intensity density (MOID) showed a significant decrease in SOD-1 and GSS ( $* \star \star ~(P<0.001), * \star(P<0.01))$ respectively), whereas MOID of NRF2, KEAP-1, and H0-1 significantly increased $(* \star \star ~(P<0.001), * \star(P<0.01)$, $\star \star \star(P<0.001)$ respectively) in aging rat, compared with young rats. However, CAT and NQO-1 remained unchanged in the two groups (no statistical significance). Abbreviations: $\mathrm{IHC}=\mathrm{Immunohistochemistry,} \mathrm{OD}=$ Optical density. 